\title{
NUMERICAL APPROXIMATION OF FIRST KIND VOLTERRA CONVOLUTION INTEGRAL EQUATIONS WITH DISCONTINUOUS KERNELS
}

\author{
PENNY J. DAVIES AND DUGALD B. DUNCAN \\ Communicated by Francisco-Javier Sayas
}

\begin{abstract}
The cubic "convolution spline" method for first kind Volterra convolution integral equations was introduced in P.J. Davies and D.B. Duncan, Convolution spline approximations of Volterra integral equations, Journal of Integral Equations and Applications 26 (2014), 369-410. Here, we analyze its stability and convergence for a broad class of piecewise smooth kernel functions and show it is stable and fourth order accurate even when the kernel function is discontinuous. Key tools include a new discrete Gronwall inequality which provides a stability bound when there are jumps in the kernel function and a new error bound obtained from a particular B-spline quasi-interpolant.
\end{abstract}

1. Introduction. In [5], we derived a new numerical method which can be used to approximate the solution $u(t)$ of the first kind Volterra integral equation (VIE)

$$
\int_{0}^{t} K(\tau) u(t-\tau) d \tau=a(t), \quad \text { for } t \in[0, T],
$$

where $a(0)=0$ and $K(0) \neq 0$, with fourth order accuracy when the convolution kernel $K$ and right-hand side $a$ are sufficiently smooth. This "convolution spline" approximation shares some properties with Lubich's convolution quadrature [11] but is explicitly constructed in terms of cubic spline basis functions. Although numerical results [5] indicate that the scheme is also fourth order convergent when $K$ is only piecewise smooth, the analysis does not extend to this case. We

2010 AMS Mathematics subject classification. Primary 65M12, 65R20.

Keywords and phrases. Volterra integral equations, discontinuous kernel, time delay.

Received by the editors on November 16, 2015, and in revised form on June 20, 2016. 
now provide a proof when $K(t)$ is piecewise smooth with (finite) jump discontinuities irrespective of where the jumps occur. In particular, convergence does not rely on fitting or adapting the stepsize so that the jumps occur at element boundaries, in contrast to the requirements of the trapezoidal rule (collocation with continuous piecewise linear approximation of $u$ ) applied to (1.1) with a step function kernel [5, subsection 4.2.2] and methods for second kind problems in, e.g., [3, Chapter 4.2] and [13].

The discontinuous kernel convolution first kind VIEs we consider are also called VIEs with constant non-vanishing delays [3, Chapter 4]. These problems are sometimes written as Volterra functional equations where initial data specifying $u(t)$ in some initial interval are given. We do not consider the functional form here since it is equivalent to a problem in the form (1.1) after a shift in the time variable and absorbing the initial data into $a(t)$.

Much of the literature on discontinuous kernel problems for VIEs concentrates on problems of the second kind. In 1911, one of the key early papers [8] described and analyzed such second kind problems. Recent numerical analysis for particular types of discontinuous second kind problems can be found in $[\mathbf{1 2}, \mathbf{1 3}]$. Collocation methods for both first and second kind VIEs with discontinuous kernels are described by Brunner [3, subsections 4.2, 4.3], and work on the analysis and numerical analysis of a different type of discontinuous kernel first kind problems appears in $[\mathbf{1 4}, \mathbf{1 8}]$. That work is for problems with proportionate, vanishing delays and does not apply to our class of problems.

Convolution quadrature methods $[\mathbf{1}, \mathbf{1 1}]$ can also be used for discontinuous kernel problems in the form (1.1). However they rely on being able to evaluate the Laplace transform of $K(t)$, which is not always straightforward, and care may be needed to evaluate the contour integrals for the weights used in the scheme when there are jumps in $K$. Our method does not use the Laplace transform of the kernel $K$ and the calculation of the weights is straightforward, with or without jumps.

Such discontinuous kernel problems arise in a variety of applications. Some first kind VIEs with a discontinuous kernel are derived in Laplace transform format in $[\mathbf{2}, \mathbf{1 7}]$. They arise as part of a separation of variables solution of a scattering problem from a circle in $2 \mathrm{D}$ and a 
sphere in 3D. For example, time-dependent acoustic scattering from a unit sphere can be decoupled into independent VIEs by expanding the incident wave into spherical harmonics, and in this case, the $n$th order spherical harmonic modes of the surface potential satisfy (1.1) with kernel

$$
K(t)=\frac{1}{2} P_{n}\left(1-\frac{1}{2} t^{2}\right) H(2-t),
$$

where $H(t)$ is the Heaviside function and $P_{n}(t)$ the degree $n$ Legendre polynomial, see [7] for details.

Another important application area is in the deconvolution of well test data from water or oil reservoirs to obtain a constant rate drawdown response function that is then used to estimate important physical properties of the reservoir. One form of this problem is given in [10, equation (4.5)]. In terms of equation (1.1), $u(t)$ is the unknown constant rate drawdown response, $K(t)$ is an actual or measured flow rate, and $a(t)$ a measured pressure change. An "ideal" well test experiment flows the well at a constant rate for a finite time and then closes the flow valve, continuing to measure the pressure change $a(t)$, so again, $K(t)$ involves a Heaviside function. More realistic tests may involve switching the flow on and off a few times, or having a generally smooth flow rate $K(t)$ with a small number of jumps. It is also common for the measured flow rate data to be interpolated by piecewise constant or linear functions. More details can be found in, e.g., [4, 9].

In order to illustrate the solution structure of equation (1.1) when the kernel is discontinuous, we consider the kernel $K(t)=1-H\left(t-T_{1}\right)$, i.e., $K=1$ for $0<t<T_{1}$ and is 0 otherwise. Taking the Laplace transform of equation (1.1), whose left-hand side is a Laplace convolution, using the notation $\bar{K}(s)=\mathcal{L}[K(t) ; s]$ gives $\bar{K}(s) \bar{u}(s)=\bar{a}(s)$, where $\bar{K}(s)=$ $\left(1-e^{-T_{1} s}\right) / s$. Thus, $\left(1-e^{-s T_{1}}\right) \bar{u}(s)=s \bar{a}(s)$, and taking the inverse transform gives the difference equation $u(t)-u\left(t-T_{1}\right)=a^{\prime}(t)$, which has solution

$$
u(t)=\sum_{k=0}^{\infty} a^{\prime}\left(t-k T_{1}\right)=\sum_{k=0}^{\left\lfloor t / T_{1}\right\rfloor} a^{\prime}\left(t-k T_{1}\right),
$$

where $\lfloor b\rfloor=$ floor $(b)$ is the largest integer less than or equal to $b$. If $a(t)$ has compact support in an interval $t \in\left[t_{L}, t_{R}\right]$ of width $t_{R}-t_{L} \leq T_{1}$, then the solution given by equation (1.2) is $T_{1}$-periodic for all $t \geq t_{L}$. 
If $a$ is localized in a region with rapid decay away from that region (but not compact support), the solution will be close to $T_{1}$-periodic. Note that the solution may also be formally obtained by writing the Laplace transform solution as

$$
\bar{u}(s)=\frac{1}{1-e^{-s T_{1}}} s \bar{a}(s)=\left(\sum_{j=0}^{\infty} e^{-s j T_{1}}\right) s \bar{a}(s),
$$

and then inverting term-by-term. The exponentials are transforms of time shift operators and $s \bar{a}(s)$ is the transform of $a^{\prime}(t)$ because $a(0)=0$.

The plan for the rest of this article is as follows. In Section 2, we derive properties of the exact solution of equation (1.1) under various assumptions on the regularity of $a$ and $K$ and also briefly describe the convolution spline approximation scheme. Section 3 contains numerical convergence results for representative benchmark problems with discontinuous kernels. Some tools needed for stability analysis are introduced in Section 4, including a new discrete Gronwall inequality for dealing with the step changes in the kernel, and we use them to establish stability of the scheme for a broad class of problems with piecewise smooth kernels. These stability results are a key step in the convergence analysis of the scheme in Section 5, and we derive a new error bound using a quasi-interpolant from the space of cubic B-splines.

\section{Preliminaries.}

2.1. Solution properties. We first determine the regularity of the solution $u$ of equation (1.1) under various assumptions on $a$ and $K$. Because $K(0) \neq 0$, we rescale the problem and will always assume that $K(0)=1$. We consider two different types of function $a$ : either

$$
a \in C^{d+1}[0, T], \quad a(0)=0 ;
$$

Or

$$
a \in C^{d+1}[0, T], \quad a^{(j)}(0)=0 \quad \text { for } j=0: d+1,
$$

for $d \geq 0$ to be specified.

Lemma 2.1 ([3, Theorem 2.1.9]). If $K(0)=1, K \in C^{d+1}[0, T]$ and condition (2.1) holds for some $d \geq 0$, then the unique solution $u$ of equation (1.1) satisfies $u \in C^{d}[0, T]$. 
We now show that the special nature of the convolution kernel allows the regularity requirement on $K$ to be relaxed, provided condition (2.2) holds.

Lemma 2.2. If $K(0)=1, K \in C^{1}[0, T]$ and condition (2.2) holds for some $d \geq 0$, then the unique solution $u$ of equation (1.1) satisfies $u \in C^{d}[0, T]$ and $u^{(p)}(0)=0$ for $p=0: d$.

Proof. The continuity of $u$ when $d=0$ is covered by Lemma 2.1 . Rewriting equation (1.1) as

$$
\int_{0}^{t} K(t-\tau) u(\tau) d \tau=a(t), \quad \text { for } t \in[0, T]
$$

and differentiating, gives

$$
u(t)+\int_{0}^{t} K^{\prime}(t-\tau) u(\tau) d \tau=a^{\prime}(t)
$$

which yields $u(0)=a^{\prime}(0)=0$.

If $d=1$, then consider the VIE

$$
\int_{0}^{\xi} K(\tau) v(\xi-\tau) d \tau=a^{\prime}(\xi)
$$

By the above, the unique solution $v$ of equation (2.4) is continuous with $v(0)=0$. Integrating equation $(2.4)$ over $(0, t)$ using $a(0)=0$ gives

$$
a(t)=\int_{0}^{t} \int_{0}^{\xi} K(\tau) v(\xi-\tau) d \tau d \xi=\int_{0}^{t} K(\tau) \int_{0}^{t-\tau} v(\xi) d \xi d \tau
$$

and comparison with equation (1.1), whose solution is unique, gives

$$
u(t)=\int_{0}^{t} v(\xi) d \xi
$$

Hence, $u \in C^{1}[0, T]$ and $u^{\prime}(0)=v(0)=0$. The result for $d \geq 2$ follows from repeating this argument $d$ times.

Note that the derivative conditions (2.2) guarantee that the extension of $u$ by 0 to the negative real axis is in $C^{d}(-\infty, T]$. If they do not hold, then any numerical approximation of equation (1.1) needs to be 
'corrected' as described for convolution quadrature in [11, Section 3] in order to attain optimal convergence.

The next result deals with the case that the kernel is piecewise smooth but discontinuous.

Lemma 2.3. Suppose that

$$
K(t)= \begin{cases}K_{0}(t) & t<T_{1} \\ K_{1}(t) & t>T_{1}\end{cases}
$$

for some $T_{1} \in(0, T)$, where $K_{0}(0)=1, K_{0} \in C^{d+1}\left[0, T_{1}\right], K_{1} \in$ $C^{d+1}\left[T_{1}, T\right]$ and, in general, $K_{0}\left(T_{1}\right) \neq K_{1}\left(T_{1}\right)$. Then, if condition (2.2) holds, the unique solution $u$ of (1.1) satisfies $u \in C^{d}[0, T]$ and $u^{(p)}(0)=$ 0 for $p=0: d$.

Proof. Applying Lemma 2.2 for $t<T_{1}$ gives

$$
u \in C^{d}\left[0, T_{1}\right] \text { and } u^{(p)}(0)=0
$$

for $p=0: d$. It remains to show that the solution $u$ extends to $[0, T]$ with no decrease in regularity, and we do this inductively by showing that the regularity can successively be extended by intervals of length $T_{1}$.

Let $\widehat{K}_{0}(t) \in C^{d+1}[0, T]$ be a smooth extension of the function $K_{0}$ to $[0, T]$, and set

$$
K_{D}(t)=\widehat{K}_{0}\left(t+T_{1}\right)-K_{1}\left(t+T_{1}\right),
$$

so $K_{D} \in C^{d+1}\left[0, T-T_{1}\right]$. As an inductive hypothesis, we assume that $u \in C^{d}\left[0, j T_{1}\right]$ for some $j \geq 1$, and we need to show that $u \in C^{d}\left[0, T_{M}\right]$, where

$$
T_{M}=\min \left\{(j+1) T_{1}, T\right\} .
$$

We rewrite equation (2.3) for $t \leq T_{M}$ as

$$
\int_{0}^{t} \widehat{K}_{0}(t-\tau) u(\tau) d \tau=a(t)+\sigma\left(t-T_{1}\right),
$$

where

$$
\sigma(t)= \begin{cases}0 & t<0, \\ \int_{0}^{t} K_{D}(t-\tau) u(\tau) d \tau & t \in\left[0, T_{M}-T_{1}\right] .\end{cases}
$$


By construction,

$$
\sigma \in C^{(d+1)}\left[0, T_{M}-T_{1}\right]
$$

$\sigma(0)=0$ and

$$
\widehat{K}_{0}(t) \in C^{d+1}[0, T],
$$

and so we only need to show that $\sigma^{(p)}(0)=0$ for $p=1: d+1$ in order to apply Lemma 2.2 and deduce that $u \in C^{d}\left[0, T_{M}\right]$. The $p$ th derivative of $\sigma(t)$ for $t \geq 0$ is

$$
\sigma^{(p)}(t)=\sum_{j=0}^{p-1} K_{D}^{(j)}(0) u^{(p-1-j)}(t)+\int_{0}^{t} K_{D}^{(p)}(t-\tau) u(\tau) d \tau,
$$

from which the required result follows at $t=0$.

We allow the kernel $K$ to have a finite number of discontinuities, at $T_{\ell}, \ell=1: N_{s}$ where

$$
0=T_{0}<T_{1}<T_{2}<\cdots<T_{N_{s}}<T_{N_{s}+1}=T,
$$

and set $K_{\ell}(t)=K(t)$ for $t \in\left(T_{\ell}, T_{\ell+1}\right)$. The arguments of Lemma 2.3 can be extended to this case, yielding the next result.

Corollary 2.4. Suppose that a satisfies condition (2.2) and

$$
K_{0}(0)=1, \quad K_{\ell} \in C^{d+1}\left(T_{\ell}, T_{\ell+1}\right) \quad \text { for } \ell=0: N_{s}
$$

for some $d \geq 0$. Then, the unique solution $u$ of equation (1.1) with $K(t)=K_{\ell}(t)$ for $t \in\left(T_{\ell}, T_{\ell+1}\right)$, satisfies $u \in C^{d}[0, T]$ and $u^{(p)}(0)=0$ for $p=0: d$.

Note that, as illustrated in Figure 1, a discontinuous kernel which satisfies condition (2.5) can be written as the sum of a continuous piecewise smooth function $K_{C}$ and $N_{s}$ constant pulse functions, i.e.,

$$
K_{C}(t):=K(t)-\sum_{\ell=1}^{N_{s}} \alpha_{\ell}\left[H\left(t-T_{\ell}\right)-H\left(t-T_{\ell+1}\right)\right],
$$

is continuous when

$$
\alpha_{0}=0 \quad \text { and } \quad \alpha_{\ell}-\alpha_{\ell-1}=K_{\ell}\left(T_{\ell}\right)-K_{\ell-1}\left(T_{\ell}\right), \quad \ell=1: N_{s}
$$


Alternatively, equation (2.6) can be written as

$$
K(t)=K_{C}(t)+\sum_{\ell=1}^{N_{s}}\left(K_{\ell}\left(T_{\ell}\right)-K_{\ell-1}\left(T_{\ell}\right)\right) H\left(t-T_{\ell}\right) .
$$

2.2. Convolution spline approximation. The convolution spline scheme [5] is a backwards-in-time approximation of the solution $u$ of equation (1.1) at time $t_{n}=n h$ with constant stepsize $h=T / N_{T}$ given by

$$
u\left(t_{n}-\tau\right) \approx U_{n}\left(t_{n}-\tau\right)=\sum_{j=0}^{n} v_{n-j} \phi_{j}(\tau / h), \quad \text { for } \tau \in\left[0, t_{n}\right],
$$

where the basis functions are cubic B-splines with a parabolic runout condition at $t=0$, that is, for $t \geq 0$,

$$
\begin{aligned}
& \left.\phi_{0}(t)=B_{3}(t)+3 B_{3}(t+1), \quad \phi_{1}(t)=B_{3}(t-1)-3 B_{3}(t+1)\right\} \\
& \left.\phi_{2}(t)=B_{3}(t-2)+B_{3}(t+1), \quad \phi_{j}(t)=B_{3}(t-j), \quad \text { for } j \geq 3\right\},
\end{aligned}
$$

where $B_{3}(t)$ is the cardinal cubic B-spline (see, e.g., a standard text such as [6]). All the basis functions $\phi_{j}$ are non-negative on $[0, \infty)$,
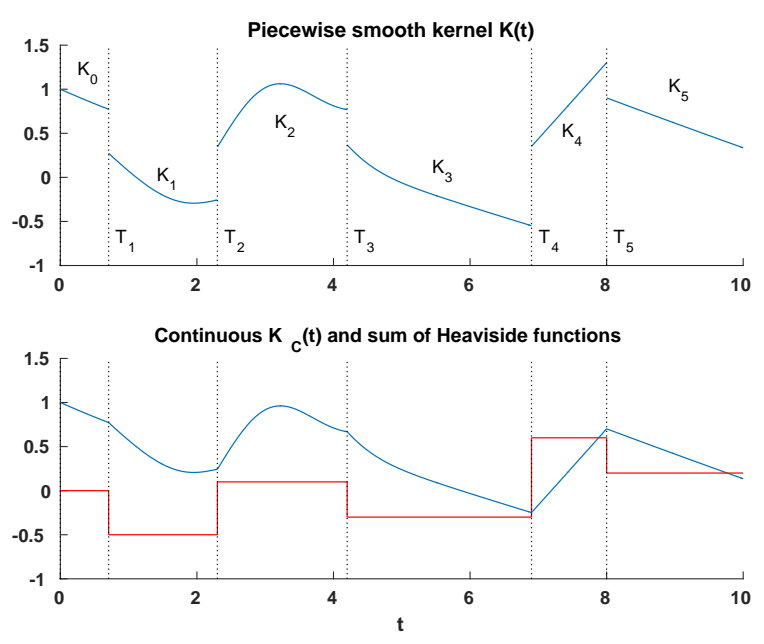

FIGURE 1. A piecewise smooth kernel function with $N_{s}=5$ discontinuities. 
except for $\phi_{1}$ which is negative for $t \in[0,1-\sqrt{2 / 3})$. The cardinal B-spline $B_{m}(t)$ for $m \geq 1$ is a positive, even function, globally $C^{m-1}$, has support in $(-(m+1) / 2,(m+1) / 2)$ and is a polynomial of degree $m$ on each interval $(k, k+1)$ for $k=-(m+1) / 2:(m-1) / 2$. It satisfies

$$
B_{m+1}^{\prime}(t)=B_{m}(t+1 / 2)-B_{m}(t-1 / 2),
$$

and integrating, gives

$$
B_{m+1}(x+1 / 2)=\int_{x}^{x+1} B_{m}(t) d t
$$

for $x>-(m+3) / 2$.

Using the fact that $u(t)=0$ for $t \leq 0$ (in other words, $u$ is causal), equation (1.1) can be written as

$$
\int_{0}^{\infty} K(\tau) u(t-\tau) d \tau=a(t), \quad t \in[0, T] .
$$

Substituting $t=t_{n}$ and the approximation (2.8) into this gives the discrete convolution equation

$$
\int_{0}^{\infty} K(\tau) U_{n}\left(t_{n}-\tau\right) d \tau=\sum_{j=0}^{n} q_{j} v_{n-j}=a\left(t_{n}\right), \quad \text { for } n=0: N_{T}
$$

for the unknown coefficients $v_{k}$, where

$$
q_{j}=\int_{0}^{\infty} K(t) \phi_{j}(t / h) d t=h \int_{\max (0, j-2)}^{j+2} K(t h) \phi_{j}(t) d t .
$$

The $v_{k}$ are obtained recursively from equation (2.11) by time marching:

$$
v_{0}=0, \quad v_{n}=\frac{1}{q_{0}}\left(a\left(t_{n}\right)-\sum_{j=0}^{n-1} q_{n-j} v_{j}\right), \quad n \geq 1 .
$$

The step size $h:=T / N_{T}$ is chosen independently of the locations $T_{\ell}$ of the jumps in $K(t)$. These locations are associated with mesh intervals by defining

$$
m_{\ell}:=\left\lfloor T_{\ell} / h\right\rfloor \in \mathbb{Z} \quad \text { and } \quad r_{\ell}:=T_{\ell} / h-m_{\ell} \in[0,1),
$$

so that

$$
T_{\ell}=\left(m_{\ell}+r_{\ell}\right) h, \quad \text { for } \ell=1: N_{s} .
$$


The case $r_{\ell}=0$ only happens if the jump location is exactly at a mesh point, and in general, $r_{\ell}>0$. For completeness, we set $m_{0}=0$, $m_{N_{s}+1}=N_{T}$ and $r_{0}=r_{N_{s}+1}=0$. We assume that step size $h$ is sufficiently small so that successive $T_{\ell}$ do not occur in intervals which are near-neighbors; in particular, we assume that

$$
m_{\ell+1}-m_{\ell} \geq 5, \quad \ell=0: N_{s}
$$

in the calculations below.

3. Benchmark problems and numerical results. Numerical results for the convolution spline approximation (2.8) of equation (1.1) for a unit step, i.e., $K(t)=1-H\left(t-T_{1}\right)$, are given in [5], and we now examine the scheme's performance on some more complicated benchmark problems. Stability and convergence results for these classes of kernels are given in Sections 4-5.

3.1. BM1: Discontinuous multiple step-function kernel. Suppose that $K$ satisfying equation (2.5) is a piecewise constant function, i.e.,

$$
K(t)=\sum_{\ell=0}^{N_{s}} \alpha_{\ell}\left[H\left(t-T_{\ell}\right)-H\left(t-T_{\ell+1}\right)\right], \quad t \in[0, T],
$$

for some $\alpha_{\ell} \in \mathbb{R}$. This can be rearranged as

$$
K(t)=1+\sum_{\ell=1}^{N_{s}}\left(\alpha_{\ell}-\alpha_{\ell-1}\right) H\left(t-T_{\ell}\right), \quad \alpha_{0}=1 .
$$

The exact solution of equation (1.1) with this kernel can again be obtained by Laplace transforms, using $\bar{K}(s)=(1-\bar{Q}(s)) / s$, where

$$
\bar{Q}(s)=\sum_{\ell=1}^{N_{s}}\left(\alpha_{\ell-1}-\alpha_{\ell}\right) e^{-s T_{\ell}} .
$$

The Laplace transform of the solution is formally obtained by writing

$$
\bar{u}(s)=(1-\bar{Q}(s))^{-1} s \bar{a}(s)=\sum_{j=0}^{\infty} \bar{Q}^{j}(s) s \bar{a}(s)
$$

in the same way as for the single step kernel example in Section 1. The function $\bar{Q}(s)$ is the transform of a linear combination of time shift 
operators, with the property

$$
\mathcal{L}^{-1}[\bar{Q}(s) s \bar{a}(s) ; t]=\mathrm{Q} a^{\prime}(t):=\sum_{\ell=1}^{N_{s}}\left(\alpha_{\ell-1}-\alpha_{\ell}\right) a^{\prime}\left(t-T_{\ell}\right),
$$

giving

$$
u(t)=\sum_{j=0}^{\left\lfloor t / T_{1}\right\rfloor} \mathrm{Q}^{j} a^{\prime}(t)
$$

Although messy to evaluate, it is possible to compute the exact solution up to any finite time, given the causal nature of $a(t)$.

Assumption (2.15) implies that the first few coefficients $q_{j}$ used in equation (2.13) are

$$
q_{j} / h= \begin{cases}5 / 8 & j=0 \\ 5 / 6 & j=1 \\ 25 / 24 & j=2 .\end{cases}
$$

For $j=m_{\ell-1}+3: m_{\ell}-2$, the coefficients are $q_{j}=\alpha_{\ell-1} h$, and, in the vicinity of the jump at $T_{\ell}$, they are

$$
q_{m_{\ell}+k} / h=\alpha_{\ell-1}+\left(\alpha_{\ell}-\alpha_{\ell-1}\right) \int_{r_{\ell}-k}^{2} B_{3}(t) d t, \quad k=-1: 2 .
$$

3.2. BM2: Piecewise smooth, globally $C^{0}$ (but not $C^{1}$ ) kernel. We consider the numerical test problem with kernel

$$
K(t)=\left[1-H\left(t-T_{1}\right)\right] \cos t,
$$

where $T_{1}=\pi / 2$. This has the Laplace transform

$$
\bar{K}(s)=\frac{s+e^{-T_{1} s}}{1+s^{2}}
$$

and, working through the formal Laplace transform procedure, eventually gives the exact solution as

$$
u(t)=\sum_{k=0}^{\left\lfloor t / T_{1}\right\rfloor}(-1)^{k} \mathcal{I}^{k+1}\left[a\left(t-k T_{1}\right)+a^{\prime \prime}\left(t-k T_{1}\right)\right],
$$

where $\mathcal{I}^{k}[f(t)]$ is the $k$ th repeated integral of $f(t)$. 

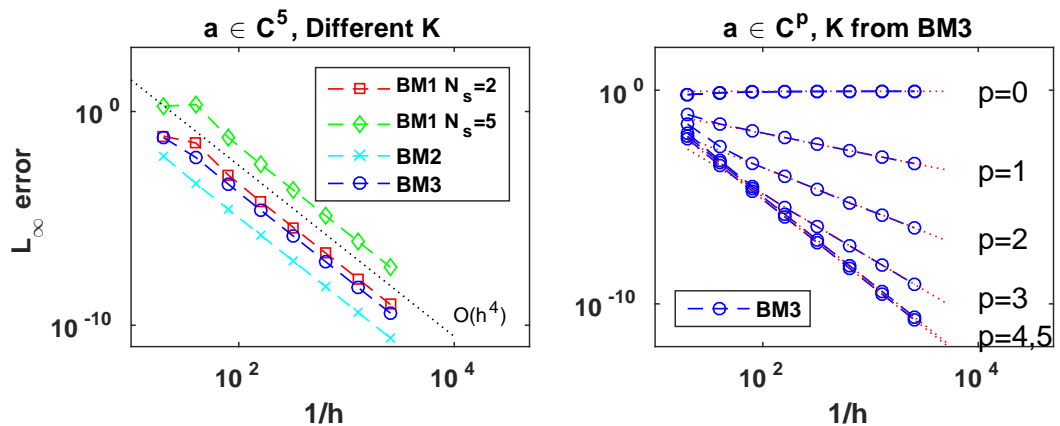

FiguRE 2. The left hand plot shows the $L_{\infty}$ error for a range of different kernel functions from the benchmark problem BM1-BM3 in subsections 3.13.3 with fixed $a \in C^{5}[0, T]$. The dotted line indicates the $\mathcal{O}\left(h^{4}\right)$ slope. The right hand plot has discontinuous kernel $K$ given in subsection 3.3 and right hand side function $a \in C^{p}[0, T]$ for $p=0: 5$. The asymptotic slopes marked are $\mathcal{O}\left(h^{p}\right)$ for $p=0: 4$.

\subsection{BM3: Discontinuous kernel, not piecewise constant.} Here, we consider

$$
K(t)=\left[1-H\left(t-T_{1}\right)\right] e^{-t},
$$

with Laplace transform

$$
\bar{K}(s)=\frac{1-e^{-T_{1}(1+s)}}{1+s} .
$$

The Laplace transformed solution formally satisfies

$$
\bar{u}(s)=\sum_{k=0}^{\infty} e^{-k T_{1}} e^{-s k T_{1}}(1+s) \bar{a}(s),
$$

giving the exact solution

$$
u(t)=\sum_{k=0}^{\left\lfloor t / T_{1}\right\rfloor} e^{-k T_{1}}\left(a\left(t-k T_{1}\right)+a^{\prime}\left(t-k T_{1}\right)\right) .
$$

Note that terms with longer delays are more heavily damped.

3.4. Numerical implementation and results. Numerical results for the benchmark problems in the previous subsections are shown in 
Figure 2. In each case, the coefficients $q_{j}$ defined by equation (2.12) are evaluated almost exactly, using high order composite Gauss quadrature over intervals of length $h$ between the nodes. If an interval contains one of the points of discontinuity $T_{\ell}$ for $\ell=1: N_{s}$, then it is split at the discontinuity and the same quadrature rule is applied on both segments. Errors in the solution are measured using the $L_{\infty}$ norm of the difference between the exact and numerical solutions at the node points, when the exact solution is available. If not, then the error is estimated by mesh halving. In all cases, the length of the interval is $T=10$, and the step size $h$ is chosen to avoid special cases in which the discontinuities occur at an integer multiple of $h$.

The plots on the left of Figure 2 all use the forcing term

$$
a(t)=t^{6} e^{-50(t-0.5)^{2}}, \quad t \geq 0,
$$

which satisfies condition (2.2) with $d=4$. The BM1 (subsection 3.1) $N_{s}=2$ case has

$$
T_{1}=1 / \sqrt{2}, \quad T_{2}=\sqrt{3 / 2} \quad \text { with } \quad \alpha_{0}=1, \alpha_{1}=0.6, \alpha_{2}=0,
$$

while the BM1 $N_{s}=5$ case has

$$
\begin{aligned}
& T_{1}=1 / \sqrt{2}, \quad T_{2}=\sqrt{3 / 2}, \\
& T_{3}=\sqrt{5 / 2}, \quad T_{4}=\sqrt{7 / 2}, \quad T_{5}=\sqrt{11 / 2}
\end{aligned}
$$

with

$$
\begin{array}{lll}
\alpha_{0}=1, & \alpha_{1}=0.6, & \alpha_{2}=-0.4, \\
\alpha_{2}=-0.1, & \alpha_{4}=0.5, & \alpha_{5}=0 .
\end{array}
$$

Problem BM2 is as described in subsection 3.2, and problem BM3 from subsection 3.3 is used with $T_{1}=1 / \sqrt{2}$. The scheme exhibits very clear $\mathcal{O}\left(h^{4}\right)$ convergence in all of these cases.

The results on the right of Figure 2 show what happens when the regularity of the forcing term $a(t)$ is reduced in problem BM3 with $T_{1}=1 / \sqrt{2}$. We use

$$
a(t)=\left(t^{6}+(p+1)^{2}(t-0.45)_{+}^{p+1}\right) e^{-50(t-0.5)^{2}}, \quad \text { for } p=0: 5,
$$

where the truncated power function is $(x)_{+}:=\max (x, 0)$ for $x \in \mathbb{R}$. If $p=0$, then $a \notin C^{1}[0, T]$, and the explicit solution $u$ given by equation (3.2) is discontinuous at each integer multiple of $T_{1}$. Figure 2 
shows that there is no convergence (in the $L_{\infty}$ norm) when $p=0$. If $p \geq 1$, then $a(t)$ satisfies condition (2.2) with $d=p-1$ and equation (3.2) gives $u \in C^{p-1}[0, T]$. The observed convergence rate is $\mathcal{O}\left(h^{\min (p, 4)}\right)$, saturating at $\mathcal{O}\left(h^{4}\right)$, which is better than might be expected for cubic spline interpolation where $u \in C^{4}$ is a standard assumption. We note that the function $u$ from equation (3.2) is smooth everywhere except at integer multiples of $T_{1}$ where its fourth derivative is discontinuous, and this special structure might be responsible for the better than expected convergence behavior.

4. Stability of the convolution spline scheme. We now describe a new technique for investigating the stability (as defined below) of approximation schemes for (1.1). The advantage of this approach over that from [5] is that it enables us to prove convergence for discontinuous kernel functions.

Definition 4.1. The approximation (2.13) of (1.1) is stable if there exists a constant $C$ independent of $h$ such that

$$
\left|v_{n}\right| \leq C, \quad \text { for } n=1: N_{T} .
$$

We first state some definitions and results which will be needed for the subsequent analysis.

4.1. Definitions and auxiliary results. We set $\|f\|=\|f\|_{L^{\infty}[0, T]}$ and define the broken norm $\|\cdot\|$ by

$$
\|f\|:=\sum_{\ell=0}^{N_{s}}\|f\|_{L^{\infty}\left(T_{\ell}, T_{\ell+1}\right)},
$$

where the points $T_{\ell}$ for $\ell=1: N_{s}$ are the allowed points of discontinuity of the kernel $K$. Note that (2.5) implies

$$
\|K\|+\left\|K^{\prime}\right\| \leq C
$$

for some constant $C$. 
Definition 4.2. The $Z$-transform of a sequence $\left\{f_{n}\right\}_{n=0}^{\infty}$ is the function $F$ given by

$$
F(\xi)=\mathcal{Z}\left\{f_{n}\right\}(\xi)=\sum_{n=0}^{\infty} f_{n} \xi^{n}
$$

where $\xi \in \mathbb{C}$ with $|\xi| \leq 1$ is such that the sum converges.

The sequence $\mu_{n}$ defined by

$$
15 \mu_{n}+5 \mu_{n-1}+5 \mu_{n-2}-\mu_{n-3}=0
$$

for $n \geq 1$ with $\mu_{0}=1$ and $\mu_{n}=0$ for $n<0$, plays a key part in the analysis, and its relevant properties are stated below.

Lemma 4.3. The $Z$-transform of the sequence $\mu_{n}$ satisfies $\mathcal{Z}\left\{\mu_{n}\right\}(\xi)=$ $1 / G_{0}(\xi)$ where

$$
G_{0}(\xi):=\left(15+5 \xi+5 \xi^{2}-\xi^{3}\right) / 15
$$

has roots $\xi_{1} \approx 6.197, \xi_{2,3} \approx-0.5986 \pm 1.4359 i$. The solution of the difference equation (4.3) is

$$
\mu_{n}=c_{1} \xi_{1}^{-n}+c_{2} \xi_{2}^{-n}+c_{3} \xi_{3}^{-n}
$$

where $c_{1} \approx 0.050, \overline{c_{3}}=c_{2} \approx 0.475-0.0897 i$, and

$$
C_{\mu}:=\sum_{j=0}^{\infty}\left|\mu_{j}\right| \approx 2.051339 .
$$

We use the standard discrete Gronwall inequality below for continuous kernel problems.

Lemma 4.4 (Discrete Gronwall inequality [16, Lemma 1.4.2]). If the sequence $x_{n} \geq 0$ satisfies

$$
x_{0} \leq a, \quad x_{n} \leq a+b \sum_{j=0}^{n-1} x_{j}, \quad \text { for } n \geq 1,
$$

for some $a, b \geq 0$, then

$$
x_{n} \leq a(1+b)^{n} \leq a e^{b n} \quad \text { for all } n \geq 0 .
$$


Discontinuous kernels whose first discontinuity is at $T_{1} \approx M h$ give rise to a stability sequence which has a localized contribution coming from $M$ steps back. The next result extends the standard Gronwall inequality bound to deal with this case.

Lemma 4.5. If the sequence $x_{n} \geq 0$ satisfies

$$
x_{n} \leq a+b \sum_{j=0}^{n-1} x_{j}+c x_{n-M} \quad \text { for } n \geq 1,
$$

with $a, b, c \geq 0$, and $x_{n} \equiv 0$ for $n<0, x_{0} \leq a$, then

$$
x_{n} \leq a(1+b)^{n}(1+c)^{\lfloor n / M\rfloor} \text { for all } n \geq 0,
$$

where $\lfloor w\rfloor$ is the largest integer less than or equal to $w \in \mathbb{R}$.

Proof. We use induction over blocks of length $M$ on the sequence $x_{n} \geq 0$ satisfying inequality (4.7), with inductive hypothesis:

$(\mathbf{I H})_{S}:$ bound (4.8) holds for $n=0: S M-1$ for some $S \geq 1$.

It follows from Lemma 4.4 that $(\mathbf{I H})_{S}$ holds when $S=1$; suppose that it is true for some $S \geq 1$. We need to show that bound (4.8) holds for $n=S M+k$ for $k=0: M-1$. For such $k$, it follows from bounds (4.7) and (4.8) that

$$
\begin{aligned}
x_{S M+k} & \leq a+a(1+c)^{S-1}\left\{c(1+b)^{(S-1) M+k}+b \sum_{j=0}^{S M-1}(1+b)^{j}\right\}+b \sum_{j=0}^{k-1} x_{S M+j} \\
& \leq a(1+c)^{S-1}\left\{c(1+b)^{(S-1) M+k}+(1+b)^{S M}\right\}+b \sum_{j=0}^{k-1} x_{S M+j} \\
& \leq a(1+c)^{S}(1+b)^{S M}+b \sum_{j=0}^{k-1} x_{S M+j} .
\end{aligned}
$$

We have thus shown that the sequence $y_{k}=x_{S M+k}$ satisfies

$$
y_{k} \leq a(1+c)^{S}(1+b)^{S M}+b \sum_{j=0}^{k-1} y_{j},
$$


and hence, it follows from Lemma 4.4 that

$$
x_{S M+k} \leq a(1+c)^{S}(1+b)^{S M}(1+b)^{k}=a(1+c)^{S}(1+b)^{S M+k},
$$

giving $(\mathbf{I H})_{S+1}$ as required.

We also need the following weighted integral mean value theorem, see e.g., [19, Theorem A.6].

Lemma 4.6. If $f$ is continuous on $[a, b]$, then, for any non-negative weight function $w$ with positive integral, there exists $\xi \in[a, b]$ such that

$$
f(\xi) \int_{a}^{b} w(x) d x=\int_{a}^{b} f(x) w(x) d x .
$$

4.2. Stability for piecewise smooth kernels. In this subsection, we begin by taking the backward difference of the approximation (2.11) and obtain bounds on the sizes of the quantities $\left(q_{j}-q_{j-1}\right) / q_{0}$ that appear, most of which are $\mathcal{O}(h)$. As noted in equation (2.6), the discontinuous kernel $K(t)$ can be written as the sum of a collection of Heaviside functions and a continuous piecewise smooth function; thus, we establish the stability of these two cases separately in subsections 4.2.1 and 4.2.2. These two results are combined to give the general case in subsection 4.2.3.

We assume that conditions (2.2) and (2.5) hold for some $d \geq 0$, and that $h$ is small enough for equation (2.15) to hold, so that the first discontinuity of $K$ occurs beyond the support of $\phi_{j}(t / h)$ for $j=0: 3$. It then follows from Lemma 4.6 that there is $\xi_{0} \in(0,2)$ with

$$
\begin{aligned}
\frac{q_{0}}{h} & =\int_{0}^{2} K(t h) \phi_{0}(t) d t \\
& =K(0) \int_{0}^{2} \phi_{0}(t) d t+h K^{\prime}\left(h \xi_{0}\right) \int_{0}^{2} t \phi_{0}(t) d t \\
& =\frac{5}{8}+\frac{31 h}{120} K^{\prime}\left(h \xi_{0}\right),
\end{aligned}
$$


and hence, $q_{0}>0$ for sufficiently small $h$. We similarly obtain

$$
\frac{q_{j}}{h}= \begin{cases}5 / 6+(59 / 60) h K^{\prime}\left(h \xi_{1}\right) & j=1, \\ 25 / 24+(241 / 120) h K^{\prime}\left(h \xi_{2}\right) & j=2, \\ 1+3 h K^{\prime}\left(h \xi_{3}\right) & j=3,\end{cases}
$$

for some $\xi_{j} \in[\max (0, j-2), j+2]$.

Taking the backward difference of equation (2.11) and dividing by $q_{0}$ gives

$$
v_{0}=0, \quad \sum_{j=0}^{n} \eta_{j} v_{n-j}=\frac{a\left(t_{n}\right)-a\left(t_{n-1}\right)}{q_{0}}, \quad n \geq 1,
$$

where

$$
\eta_{0}:=1, \quad \eta_{j}:=\frac{q_{j}-q_{j-1}}{q_{0}}, \quad j \geq 1 .
$$

It follows from condition (2.2) and the above calculations that

$$
\frac{\left|a\left(t_{n}\right)-a\left(t_{n-1}\right)\right|}{q_{0}} \leq \frac{8 a_{\Delta} / 5}{1+(31 / 75) h K^{\prime}\left(h \xi_{0}\right)}, \quad n=1: N_{T},
$$

where

$$
a_{\Delta}:=\max _{n \leq N_{T}}\left\{\frac{\left|a\left(t_{n}\right)-a\left(t_{n-1}\right)\right|}{h}\right\},
$$

and the leading coefficients in equation (4.10) are

$$
\eta_{1}=1 / 3+\eta_{1}^{*}, \quad \eta_{2}=1 / 3+\eta_{2}^{*}, \quad \eta_{3}=-1 / 15+\eta_{3}^{*},
$$

where $\left|\eta_{j}^{*}\right| \leq 2 h \max \left\{\left|K^{\prime}(h \xi)\right|: \xi \in[0,5]\right\}$ for $j=1: 3$ when $h$ is sufficiently small. It is also straightforward to verify that, if $K(t)$ is continuous for $t \in\left[t_{j-3}, t_{j+2}\right], j \geq 4$, then

$$
\left|q_{j}-q_{j-1}\right| \leq h^{2}\left\|K^{\prime}\right\|
$$

(and $\left|\eta_{j}\right| \leq\left(h^{2} / q_{0}\right)\left\|K^{\prime}\right\| \leq 2 h\left\|K^{\prime}\right\|$ for sufficiently small $h$ ), but, if $K$ is discontinuous at $t=T_{\ell} \in\left[t_{j-3}, t_{j+2}\right]$, then $\eta_{j}$ is an $\mathcal{O}(1)$ quantity.

4.2.1. Discontinuous multiple step-function kernel. Stability results for the case of a single jump step-function kernel were obtained in [5], but the modified Gronwall lemma (Lemma 4.5) introduced above 
allows us to obtain a sharper result, as well as treating the more difficult case of multiple jumps.

If the kernel $K$ is given by equation (3.1) with $\alpha_{0}=1$, then the coefficients $\eta_{j}$ are

$$
\begin{aligned}
& \eta_{1}=\eta_{2}=1 / 3, \\
& \eta_{3}=-1 / 15
\end{aligned}
$$

and

$$
\eta_{j}=0,
$$

for $j=m_{\ell-1}+4: m_{\ell}-2$ for each $\ell$. The values around the jump discontinuity at $T_{\ell}$ are

$$
\begin{aligned}
\eta_{m_{\ell}+k} & =\frac{8}{5}\left(\alpha_{\ell}-\alpha_{\ell-1}\right) \int_{r_{\ell}-k}^{r_{\ell}-k+1} B_{3}(t) d t \\
& =\frac{8}{5}\left(\alpha_{\ell}-\alpha_{\ell-1}\right) \beta_{k}(r),
\end{aligned}
$$

for $k=-1: 3$, using equation (2.10), where $\beta_{k}(r)=B_{4}\left(r_{\ell}-k+1 / 2\right) \geq$ 0 . Substituting the values of $\eta_{j}$ into equation (4.10) gives

$$
\begin{aligned}
\frac{15 v_{n}+}{} & 5 v_{n-1}+5 v_{n-2}-v_{n-3} \\
= & \frac{8}{5}\left\{\frac{a\left(t_{n}\right)-a\left(t_{n-1}\right)}{h}+\sum_{\ell=1}^{N_{s}}\left(\alpha_{\ell-1}-\alpha_{\ell}\right) \sum_{k=-1}^{3} \beta_{k} v_{n-m_{\ell}-k}\right\}
\end{aligned}
$$

with $v_{0}=0$ for $k \leq 0$. The $Z$-transform of this difference scheme is

$$
G_{0}(\xi) V(\xi)=\frac{8}{5}\left\{\frac{(1-\xi) A(\xi)}{h}+\sum_{\ell=1}^{N_{s}}\left(\alpha_{\ell-1}-\alpha_{\ell}\right) \sum_{k=-1}^{3} \beta_{k} \xi^{m_{\ell}+k} V(\xi)\right\},
$$

where $G_{0}$ is defined in equation (4.4). Using Lemma 4.3 and taking the inverse transform gives

$$
v_{n}=\frac{8}{5} \sum_{j=0}^{n} \mu_{n-j}\left\{\frac{a\left(t_{j}\right)-a\left(t_{j-1}\right)}{h}+\sum_{\ell=1}^{N_{s}}\left(\alpha_{\ell-1}-\alpha_{\ell}\right) \sum_{k=-1}^{3} \beta_{k} v_{j-m_{\ell}-k}\right\},
$$


and it follows from equation (4.6) and bound (4.12) that

$$
\left|v_{n}\right| \leq \frac{8}{5} C_{\mu} a_{\Delta}+\frac{8}{5} \sum_{j=0}^{n}\left|\mu_{n-j}\right| \sum_{\ell=1}^{N_{s}}\left|\alpha_{\ell-1}-\alpha_{\ell}\right| \sum_{k=-1}^{3} \beta_{k}(r)\left|v_{j-m_{\ell}-k}\right|,
$$

for $n \geq 1$. To make further progress with this inequality we introduce the cumulative maximum modulus:

$$
z_{n}:=\max _{0 \leq j \leq n}\left|v_{j}\right|, \quad n>0
$$

with $z_{n}=0$ for all $n \leq 0$. Then, the second term on the right-hand side of the term (4.14) can be bounded by:

$$
\begin{aligned}
\frac{8}{5} \sum_{j=0}^{n}\left|\mu_{n-j}\right| z_{n-m_{1}+1} \sum_{\ell=1}^{N_{s}} \mid & \alpha_{\ell-1}-\alpha_{\ell} \mid \sum_{k=-1}^{3} \beta_{k}(r) \\
& =\frac{8}{5} z_{n-m_{1}+1} \sum_{j=0}^{n}\left|\mu_{n-j}\right| \sum_{\ell=1}^{N_{s}}\left|\alpha_{\ell-1}-\alpha_{\ell}\right|
\end{aligned}
$$

since $\sum_{k=-1}^{3} \beta_{k}(r)=1$ for all $r \in[0,1)$ from the properties of quartic splines. Hence,

$$
\left|v_{n}\right| \leq C_{1}+C_{2} z_{n-m_{1}+1}
$$

for each $n \geq 0$, where $C_{1}=8 C_{\mu} a_{\Delta} / 5, a_{\Delta}$ is defined in equation (4.13) and

$$
C_{2}=8 C_{\mu} \sum_{\ell=1}^{N_{s}}\left|\alpha_{\ell-1}-\alpha_{\ell}\right| / 5
$$

If $0 \leq k \leq n$, then

$$
\left|v_{k}\right| \leq C_{1}+C_{2} z_{k-m_{1}+1} \leq C_{1}+C_{2} z_{n-m_{1}+1}
$$

and so,

$$
z_{n} \leq C_{1}+C_{2} z_{n-m_{1}+1}
$$

Finally, applying the modified Gronwall inequality, Lemma 4.5 gives the stability bound

$$
\left|v_{n}\right| \leq z_{n} \leq C_{1}\left(1+C_{2}\right)^{\left\lfloor n /\left(m_{1}-1\right)\right\rfloor},
$$


for $n=1: N_{T}$. Note that

$$
\left\lfloor n /\left(m_{1}-1\right)\right\rfloor \leq T /\left(T_{1}-2 h\right),
$$

and so, $\left|v_{n}\right|$ is bounded independently of $h$.

4.2.2. Continuous piecewise $C^{1}$ kernels. The convergence proof of [5] (which implies stability) needs $a, K \in C^{7}[0, T]$, but, as shown below, far less regularity is needed. We assume that $K$ is globally continuous on $[0, T]$ and that $a$ and $K$, respectively, satisfy conditions (2.2) and (2.5) with $d=0$.

The scheme (4.10) can be rewritten as

$$
\frac{15 v_{n}+5 v_{n-1}+5 v_{n-2}-v_{n-3}}{15}=\frac{a\left(t_{n}\right)-a\left(t_{n-1}\right)}{q_{0}}-\sum_{j=0}^{n-1} \eta_{n-j}^{*} v_{j},
$$

where $\eta_{0}^{*}=0, \eta_{j}^{*}$ for $j=1: 3$ are as defined in Section 4.2 and $\eta_{j}^{*}=\eta_{j}$ for $j \geq 4$. The bounds from subsection 4.2 give

$$
\left|\eta_{j}^{*}\right| \leq 2 h\left\|K^{\prime}\right\|
$$

for each $j$. As in the previous subsection, taking the $Z$-transform of the above difference scheme gives

$$
G_{0}(\xi) V(\xi)=\frac{(1-\xi) A(\xi)}{q_{0}}-\mathcal{Z}\left\{\eta_{n}^{*}\right\}(\xi) V(\xi),
$$

and we again use Lemma 4.3 and take the inverse transform to obtain

$$
V(\xi)=\mathcal{Z}\left\{\mu_{n}\right\}(\xi)\left\{\frac{(1-\xi) A(\xi)}{q_{0}}-\mathcal{Z}\left\{\eta_{n}^{*}\right\}(\xi) V(\xi)\right\}
$$

and

$$
v_{n}=\sum_{j=0}^{n} \mu_{n-j}\left(\frac{a\left(t_{j}\right)-a\left(t_{j-1}\right)}{q_{0}}\right)-\sum_{j=0}^{n} \mu_{n-j} \sum_{k=0}^{j-1} \eta_{j-k}^{*} v_{k}, \quad n \geq 1 .
$$

It then follows from Lemma 4.3 and the bounds of subsection 4.2 that

$$
\left|v_{n}\right| \leq 2 C_{\mu} a_{\Delta}+9 C_{\mu}\left\|K^{\prime}\right\| h \sum_{j=0}^{n-1}\left|v_{j}\right|
$$


for $n=1: N_{T}$. The standard Gronwall inequality in Lemma 4.4 then gives the stability result

$$
\left|v_{n}\right| \leq 2 C_{\mu} a_{\Delta} \exp \left(2 C_{\mu}\left\|K^{\prime}\right\| n h\right) \leq 2 C_{\mu} a_{\Delta} \exp \left(2 C_{\mu}\left\|K^{\prime}\right\| T\right),
$$

for $n=1: N_{T}$ where $a_{\Delta}$ is defined in equation (4.13).

4.2.3. General piecewise $C^{1}$ kernel. The results of the previous two subsections are now combined to prove the next result.

Theorem 4.7. Suppose that conditions (2.2) and (2.5) hold for $d=0$. Then, for sufficiently small $h$, the solution $v_{n}$ of equation (4.10) satisfies

$$
\left|v_{n}\right| \leq C_{1} e^{C_{2} T}\left(1+C_{3}\right)^{\left\lfloor n /\left(m_{1}-1\right)\right\rfloor},
$$

for $n=1: N_{T}$, where

$$
\begin{aligned}
& C_{1}:=2 C_{\mu} a_{\Delta}, \\
& C_{2}:=2 C_{\mu}\left\|K^{\prime}\right\|, \\
& C_{3}:=2 C_{\mu} \sum_{\ell=1}^{N_{s}}\left|K_{\ell-1}\left(T_{\ell}\right)-K_{\ell}\left(T_{\ell}\right)\right|,
\end{aligned}
$$

$C_{\mu}$ given by equation (4.6) and $a_{\Delta} \leq\left\|a^{\prime}\right\|$ is defined in equation (4.13).

Proof. As in equation (2.6), we write $K$ as the sum of a continuous piecewise $C^{1}$ function $K_{C}$ and piecewise constant functions:

$$
K(t)=K_{C}(t)+\sum_{\ell=1}^{N_{s}} \alpha_{\ell}\left[H\left(t-T_{\ell}\right)-H\left(t-T_{\ell+1}\right)\right]
$$

where the $\alpha_{\ell}$ are as defined in equation (2.7). We use the results of the previous two subsections to split the coefficients $\eta_{j}$ into two parts:

$$
\eta_{j}=\eta_{j}^{\dagger}+\eta_{j}^{*}
$$

where the $\eta_{j}^{\dagger}$ terms correspond to the piecewise constant parts (see subsection 4.2.1) and are given by

$$
\begin{aligned}
& \eta_{0}^{\dagger}=1, \\
& \eta_{1}^{\dagger}=\eta_{2}^{\dagger}=\frac{1}{3},
\end{aligned}
$$




$$
\eta_{3}^{\dagger}=-\frac{1}{15}
$$

and

$$
\eta_{j}^{\dagger}=0 \quad \text { for } j=m_{\ell-1}+4: m_{\ell}-2,
$$

for each $\ell$. The values around the jump discontinuity at $T_{\ell}$ are

$$
\eta_{m_{\ell}+k}^{\dagger}=\frac{h\left(\alpha_{\ell}-\alpha_{\ell-1}\right)}{q_{0}} \beta_{k}(r), \quad \text { for } k=-1: 3 .
$$

As in the previous subsection, the remainder terms $\eta_{j}^{*}$ satisfy

$$
\eta_{0}^{*}=0, \quad\left|\eta_{j}^{*}\right| \leq 2 h\left\|K^{\prime}\right\|
$$

Scheme (4.10) can be thus be written as

$$
\begin{aligned}
\frac{15 v_{n}+5\left(v_{n-1}+v_{n-2}\right)-v_{n-3}}{15}= & \frac{a\left(t_{n}\right)-a\left(t_{n-1}\right)}{q_{0}}-\sum_{j=0}^{n-1} \eta_{n-j}^{*} v_{j} \\
& +\frac{h}{q_{0}} \sum_{\ell=1}^{N_{s}}\left(\alpha_{\ell-1}-\alpha_{\ell}\right) \sum_{k=-1}^{3} \beta_{k} v_{n-m_{\ell}-k} .
\end{aligned}
$$

We again take the $Z$-transform, using Lemma 4.3 and the inverse transform to obtain

$$
\begin{aligned}
v_{n}= & \sum_{j=0}^{n} \mu_{n-j}\left(\frac{a\left(t_{j}\right)-a\left(t_{j-1}\right)}{q_{0}}\right)-\sum_{j=0}^{n} \mu_{n-j} \sum_{k=0}^{j-1} \eta_{j-k}^{*} v_{k} \\
& +\frac{h}{q_{0}} \sum_{\ell=1}^{N_{s}}\left(\alpha_{\ell-1}-\alpha_{\ell}\right) \sum_{k=-1}^{3} \beta_{k} v_{j-m_{\ell}-k},
\end{aligned}
$$

which gives the bound

$$
\left|v_{n}\right| \leq C_{1}+C_{2} h \sum_{j=0}^{n-1}\left|v_{j}\right|+C_{3} z_{n-m_{1}+1},
$$

for $n \geq 1$, where $z_{n}$ is the cumulative maximum defined in equation (4.15) and the constants $C_{i}$ are given by equation (4.17). Note that $C_{3}$ is obtained because

$$
\left|\alpha_{\ell}-\alpha_{\ell-1}\right|=\left|K_{\ell}\left(T_{\ell}\right)-K_{\ell-1}\left(T_{\ell}\right)\right|
$$


If $k \leq n$, then

$$
\left|v_{k}\right| \leq C_{1}+C_{2} h \sum_{j=0}^{n-1} z_{j}+C_{3} z_{n-m_{1}+1}
$$

giving

$$
z_{n} \leq C_{1}+C_{2} h \sum_{j=0}^{n-1} z_{j}+C_{3} z_{n-m_{1}+1} .
$$

Finally, we use the modified Gronwall Lemma 4.5 to obtain

$$
z_{n} \leq C_{1}\left(1+C_{2} h\right)^{n}\left(1+C_{3}\right)^{\left\lfloor n /\left(m_{1}-1\right)\right\rfloor},
$$

giving equation (4.16) as required.

5. Convergence. We show next that, under reasonable hypotheses and for a wide range of kernel functions, the difference between the exact solution $u$ of (1.1) and its convolution spline approximation $U_{n}(t)$ satisfies

$$
\left|U_{n}(t)-u(t)\right| \leq \begin{cases}C h^{4} & 0 \leq t \leq t_{n-1} \\ C h^{3} & t_{n-1}<t \leq t_{n}\end{cases}
$$

for $n=1: N_{T}$. This is achieved by introducing a quasi-interpolant $\widehat{U}(t)$ from the cubic B-spline space and showing that it is within $\mathcal{O}\left(h^{4}\right)$ of the exact solution, and within $\mathcal{O}\left(h^{4}\right)$ of the approximate solution over most of the range.

For technical reasons, we need $u(t) \in C^{4}[-2 h, T+2 h]$, and so we extend the definition of $K(t)$ and $a(t)$ for $t$ up to $T+2 h$. The maximum norm taken over the range $[0, T+2 h]$ is denoted by an asterisk, i.e.,

$$
\|\cdot\|_{*}=\|\cdot\|_{L_{\infty}[0, T+2 h]} \cdot
$$

5.1. A quasi-interpolant of $u(t)$. We assume that $u \in C^{4}[0, T+2 h]$ with $u^{(p)}(0)=0$ for $p=0: 4$ (Lemmas 2.2 and 2.3 give sufficient conditions on $a$ and $K$ for this). The extension of $u$ by 0 to the negative real axis is in $C^{4}[-2 h, T+2 h]$, and

$$
\left\|u^{(p)}\right\|_{L_{\infty}[-2 h, T+2 h]}=\left\|u^{(p)}\right\|_{*} \quad \text { for } p=0: 4 .
$$


Following Powell [15, Chapter 20.4], we define the quasi-interpolant $\widehat{U}$ of $u$ by

$$
\widehat{U}(t):=\sum_{j=0}^{N_{T}+1} \widehat{u}_{j} B_{3}(t / h-j), \quad t \in \mathbb{R}
$$

with coefficients

$$
\widehat{u}_{j}=\frac{4}{3} u\left(t_{j}\right)-\frac{1}{6}\left(u\left(t_{j-1}\right)+u\left(t_{j+1}\right)\right), \quad j=0: N_{T}+1 .
$$

The function $\widehat{U}(t)$ has compact support with

$$
\widehat{U}(t)=0, \quad t \notin(-2 h, T+3 h),
$$

and its approximation error is given in the next lemma.

Lemma 5.1. Given $u \in C^{4}[-2 h, T+2 h]$ with $u(t) \equiv 0$ for $t \leq 0$, then $\widehat{U}$, defined by equation (5.1), satisfies

$$
\|\widehat{U}-u\|_{L_{\infty}[-2 h, T]} \leq \frac{35 h^{4}}{1152}\left\|u^{(4)}\right\|_{*} .
$$

Proof. This follows results in [15, Chapters 20.4, 22.4] by rewriting $\widehat{U}(t)$ in each interval $t_{j} \leq t \leq t_{j+1}$, for $j=-2: N_{T}-1$, as

$$
\widehat{U}\left(t_{j}+s h\right)=\sum_{k=-2}^{3} u\left(t_{j+k}\right) b(s-k), \quad s \in[0,1]
$$

where

$$
b(s):=\frac{\left(8 B_{3}(s)-B_{3}(s+1)-B_{3}(s-1)\right)}{6} .
$$

Standard $B$-spline properties show that $b(s)$ has compact support in $(-3,3)$ and

$$
\sum_{k=-\infty}^{\infty} k^{m} b(s-k)=s^{m} \quad \text { for } m=0: 3 .
$$


Fix $j \leq N_{T}-1$ and $t=t_{j}+s h \in\left[t_{j}, t_{j+1}\right]$, and let $\mathrm{L}_{j}: C[-2 h, T+2 h] \rightarrow$ $\mathbb{R}$ be the linear functional defined by

$$
\mathrm{L}_{j}[f]=f\left(t_{j}+s h\right)-\sum_{k=-2}^{3} f\left(t_{j+k}\right) b(s-k) .
$$

Using identity (5.4) to verify that $\mathrm{L}_{j}$ annihilates cubic polynomials is straightforward, and it follows from the Peano kernel theorem that

$$
u\left(t_{j}+s h\right)-\widehat{U}\left(t_{j}+s h\right)=\int_{t_{j-2}}^{t_{j+3}} P_{K}(\theta, s) u^{(4)}(\theta) d \theta
$$

where

$$
P_{K}(\theta, s):=\frac{1}{3 !}\left(\left(t_{j}+s h-\theta\right)_{+}^{3}-\sum_{k=-2}^{3} b(s-k)\left(t_{j+k}-\theta\right)_{+}^{3}\right),
$$

and $(x)_{+}$is the truncated power term from subsection 3.4. By definition, $P_{K}(\theta, s)=0$ for $\theta \notin\left(t_{j-2}, t_{j+3}\right)$, and it can be shown that $P_{K}(\theta, s) \geq 0$ for $\theta \in\left(t_{j-2}, t_{j+3}\right)$, e.g., by considering each of the intervals $\left(t_{j}, t_{j}+s h\right),\left(t_{j}+s h, t_{j+1}\right)$ and $\left(t_{j+k}, t_{j+k+1}\right)$ for $k=-2,-1,1,2$ separately. Hence, the integral mean value theorem (Lemma 4.6) can be applied and

$$
\begin{aligned}
u\left(t_{j}+s h\right)-\widehat{U}\left(t_{j}+s h\right) & =u^{(4)}\left(\zeta_{j}\right) \int_{t_{j-2}}^{t_{j+3}} P_{K}(\theta, s) d \theta \\
& =u^{(4)}\left(\zeta_{j}\right) \frac{h^{4}}{72}\left(2+3 s^{2}-6 s^{3}+3 s^{4}\right),
\end{aligned}
$$

for some $\zeta_{j} \in\left(t_{j-2}, t_{j+3}\right)$. The polynomial in $s$ is positive with maximum value $35 / 1152$, and so,

$$
\left|u\left(t_{j}+s h\right)-\widehat{U}\left(t_{j}+s h\right)\right| \leq \frac{35 h^{4}}{1152}\left|u^{(4)}\left(\zeta_{j}\right)\right| \leq \frac{35 h^{4}}{1152}\left\|u^{(4)}\right\|_{*},
$$

and the result follows.

5.2. The difference between the approximate solution and the quasi-interpolant. Because the exact solution $u(t)$ of equation (1.1) is 0 for $t \leq 0$, equation (2.11) gives

$$
\int_{0}^{\infty} K(t) u\left(t_{n}-t\right) d t=a\left(t_{n}\right)=\int_{0}^{\infty} K(t) U_{n}\left(t_{n}-t\right) d t
$$


for $n=1: N_{T}$, and so,

$$
\begin{aligned}
R_{n}^{2} & :=\int_{0}^{\infty} K(t)\left(u\left(t_{n}-t\right)-\widehat{U}\left(t_{n}-t\right)\right) d t \\
& =\int_{0}^{\infty} K(t)\left(U_{n}\left(t_{n}-t\right)-\widehat{U}\left(t_{n}-t\right)\right) d t
\end{aligned}
$$

for $n=1: N_{T}$. It follows from approximation (2.8) and equation (5.1) that, if $t \in\left[0, t_{n}\right]$, then

$$
\begin{aligned}
U_{n}\left(t_{n}-t\right)-\widehat{U}\left(t_{n}-t\right)= & \sum_{j=0}^{n} v_{n-j} \phi_{j}(t / h)-\sum_{j=-1}^{n} \widehat{u}_{n-j} B_{3}(t / h-j) \\
= & \sum_{j=0}^{n} \varepsilon_{n-j} \phi_{j}(t / h) \\
& -\left(\widehat{u}_{n+1}-3 \widehat{u}_{n}+3 \widehat{u}_{n-1}-\widehat{u}_{n-2}\right) B_{3}\left(\frac{t}{h}+1\right)
\end{aligned}
$$

where $\varepsilon_{j}:=v_{j}-\widehat{u}_{j}$ are the nodal errors. Substituting this into equation (5.5) then gives

$$
\sum_{j=0}^{n} q_{j} \varepsilon_{n-j}=R_{n}^{1}+R_{n}^{2}, \quad n=1: N_{T},
$$

where $R_{n}^{2}$ is defined above and

$$
R_{n}^{1}:=\left(\widehat{u}_{n+1}-3 \widehat{u}_{n}+3 \widehat{u}_{n-1}-\widehat{u}_{n-2}\right) \int_{0}^{\infty} K(t) B_{3}\left(\frac{t}{h}+1\right) d t
$$

The nodal error equation (5.7) has the same coefficients as the approximation scheme (2.11),

$$
\sum_{j=0}^{n} q_{j} v_{n-j}=a\left(t_{n}\right)
$$

and thus, we can apply Theorem 4.7 with $R^{1}{ }_{n}+R^{2}{ }_{n}$ in place of $a\left(t_{n}\right)$ to obtain the following result. 
Lemma 5.2. Suppose that (2.5) holds for $d \geq 0$. Then, if $h$ is sufficiently small,

$$
\max _{0 \leq j \leq N_{T}}\left|\varepsilon_{j}\right| \leq C_{A} \max _{1 \leq n \leq N_{T}} \frac{\left|R^{1}{ }_{n}-R^{1}{ }_{n-1}+R_{n}^{2}-R^{2}{ }_{n-1}\right|}{h} .
$$

where $C_{A}:=2 C_{\mu} e^{C_{2} T}\left(1+C_{3}\right)^{\left(1+T / T_{1}\right)}$ for constants $C_{\mu}, C_{2}$ and $C_{3}$ as defined in Theorem 4.7 .

We now show that if the exact solution $u$ of equation (1.1) is sufficiently smooth, then the difference of the residuals is $\mathcal{O}\left(h^{5}\right)$.

Lemma 5.3. Suppose that the kernel $K(t)$ and right-hand side $a(t)$ of equation (1.1) satisfy conditions (2.5) and (2.2), respectively, with $d=4$ for $t \in[0, T+2 h]$. Then, if $h$ is sufficiently small, the residuals $R^{1}{ }_{n}$ and $R^{2}{ }_{n}$ defined by equations (5.8) and (5.5) satisfy

$$
\begin{aligned}
& \left|R_{n}^{1}-R_{n-1}^{1}\right| \leq \frac{h^{5}}{12}\left\|u^{(4)}\right\|_{*} \\
& \left|R_{n}^{2}-R_{n-1}^{2}\right| \leq C_{B} h^{5}\left\|u^{(4)}\right\|_{*}
\end{aligned}
$$

where

$$
C_{B}=\frac{35}{1152}\left(T\left\|K^{\prime}\right\|+\sum_{\ell=0}^{N_{s}}\left|K\left(T_{\ell}^{-}\right)-K\left(T_{\ell}^{+}\right)\right|+2 h\left\|K^{\prime}\right\|_{L_{\infty}[T, T+2 h]}\right) .
$$

Proof. It follows from the integral mean value theorem equation (4.9) that

$$
\int_{0}^{\infty} K(t) B_{3}\left(\frac{t}{h}+1\right) d t=\frac{h}{6} \int_{0}^{1}(1-s)^{3} K(s h) d s=\frac{h}{24} K(h \xi),
$$

for some $\xi \in(0,1)$, and taking the difference of $R^{1}{ }_{n}$ defined in equation (5.8) then gives

$$
R_{n}^{1}-R_{n-1}^{1}=\frac{h K(h \xi)}{24}\left(\widehat{u}_{n+1}-4 \widehat{u}_{n}+6 \widehat{u}_{n-1}-4 \widehat{u}_{n-2}+\widehat{u}_{n-3}\right) .
$$

It was shown in Corollary 2.4 that the given hypotheses on $K$ and $a$ give $u \in C^{4}[-2 h, T+2 h]$, and any $C^{4}$ function $f$ satisfies the identity $f\left(t_{n+2}\right)-4 f\left(t_{n+1}\right)+6 f\left(t_{n}\right)-4 f\left(t_{n-1}\right)+f\left(t_{n-2}\right)=h^{4} \int_{-2}^{2} B_{3}(s) f^{(4)}\left(t_{n}+s h\right) d s$, 
see, e.g., [15, Theorem 22.3]). Rearranging the definition (5.2) of $\widehat{u}_{n}$ thus gives

$$
\widehat{u}_{n+1}-4 \widehat{u}_{n}+6 \widehat{u}_{n-1}-4 \widehat{u}_{n-2}+\widehat{u}_{n-3}=h^{4} \int_{-3}^{3} b(s) u^{(4)}\left(t_{n-1}+s h\right) d s,
$$

for $b(s)$ defined in (5.3), and so

$$
R_{n}^{1}-R_{n-1}^{1}=\frac{h^{5} K(h \xi)}{24} \int_{-3}^{3} b(s) u^{(4)}\left(t_{n-1}+s h\right) d s .
$$

Because $b(s)$ takes both positive and negative values, the integral mean value theorem cannot be used directly, but it can be used after taking the modulus. We have

$$
\int_{-3}^{3}|b(s)| d s=\frac{4222+84 \times 18^{1 / 3}+25 \times 18^{2 / 3}}{3993}=1.15548 \ldots,
$$

which gives the bound (5.9) for sufficiently small $h$ (because $K(0)=1$ ).

In order to bound $R^{2}{ }_{n}-R_{n-1}^{2}$, note that

$$
R_{n}^{2}=\int_{-2 h}^{t_{n}} K\left(t_{n}-t\right)(u(t)-\widehat{U}(t)) d t
$$

taking into account the causality of the exact solution, $u(t)=0$ for $t \leq 0$, and the compact support of $\widehat{U}(t)$. Then

$$
R_{n}^{2}-R_{n-1}^{2}=\int_{-2 h}^{t_{n}}\left(K\left(t_{n}-t\right)-K\left(t_{n-1}-t\right)\right)(u(t)-\widehat{U}(t)) d t,
$$

where, for convenience, we extend $K(t)$ by zero for $t<0$. Hence,

$$
\begin{aligned}
\left|R_{n}^{2}-R_{n-1}^{2}\right| & \leq\|\widehat{U}-u\|_{L_{\infty}[-2 h, T]} \int_{-2 h}^{t_{n}}\left|K\left(t_{n}-t\right)-K\left(t_{n-1}-t\right)\right| d t \\
& \leq \frac{35 h^{4}}{1152}\left\|u^{(4)}\right\| \int_{0}^{t_{n+2}}|K(t)-K(t-h)| d t
\end{aligned}
$$

using Lemma 5.1. The bound (5.10) then follows from

$$
\begin{gathered}
\int_{T_{\ell}}^{T_{\ell+1}}|K(t)-K(t-h)| d t=\int_{T_{\ell}}^{T_{\ell}+h}|K(t)-K(t-h)| d t \\
+\int_{T_{\ell}+h}^{T_{\ell+1}}|K(t)-K(t-h)| d t
\end{gathered}
$$




$$
\leq h\left|K\left(T_{\ell}^{-}\right)-K\left(T_{\ell}^{+}\right)\right|+h^{2}\left\|K^{\prime}\right\|+\left(T_{\ell+1}-T_{\ell}-h\right) h\left\|K^{\prime}\right\| .
$$

Combining these lemmas yields our final convergence result.

Theorem 5.4. Suppose that $K$ and a satisfy the hypotheses of Lemma 5.3. Then, for sufficiently small $h$, for each $n=1: N_{T}$, the approximate solution $U_{n}(t)$ for $t \in\left[0, t_{n}\right]$ given by equation (2.11) satisfies

$$
\left|U_{n}(t)-u(t)\right| \leq C_{E}\left\|u^{(4)}\right\|_{*} h^{4}+C_{F}\left\|u^{(3)}\right\|_{*} B_{3}(t / h-n-1) h^{3}
$$

where

$$
\begin{aligned}
C_{E} & :=\frac{5}{3} C_{A}\left\{\frac{1}{12}+C_{B}\right\}+\frac{35}{1152}, \\
C_{F} & :=\frac{516+11 \sqrt{11}}{450}
\end{aligned}
$$

for $C_{A}$ and $C_{B}$ as defined in Lemmas 5.2-5.3, that is,

$$
\left|U_{n}(t)-u(t)\right| \leq \begin{cases}C_{E}\left\|u^{(4)}\right\|_{*} h^{4} & 0 \leq t \leq t_{n-1} \\ C_{F}\left\|u^{(3)}\right\|_{*} h^{3} / 6+\mathcal{O}\left(h^{4}\right) & t_{n-1}<t \leq t_{n} .\end{cases}
$$

Proof. We prove the result by adding and subtracting the quasiinterpolant $\widehat{U}$. For $t \in\left[0, t_{n}\right]$,

$$
\begin{aligned}
\left|U_{n}\left(t_{n}-t\right)-u\left(t_{n}-t\right)\right| \leq & \left|U_{n}\left(t_{n}-t\right)-\widehat{U}\left(t_{n}-t\right)\right|+\left|\widehat{U}\left(t_{n}-t\right)-u\left(t_{n}-t\right)\right| \\
= & \left|\sum_{j=0}^{n} \varepsilon_{n-j} \phi_{j}(t / h)-R_{n} B_{3}(t / h+1)\right| \\
& +\left|\widehat{U}\left(t_{n}-t\right)-u\left(t_{n}-t\right)\right| \\
\leq & \sum_{j=0}^{n}\left|\varepsilon_{n-j}\right|\left|\phi_{j}(t / h)\right|+\left|R_{n}\right| B_{3}(t / h+1) \\
& +\left|\widehat{U}\left(t_{n}-t\right)-u\left(t_{n}-t\right)\right|
\end{aligned}
$$

using equation (5.6), where

$$
R_{n}=\widehat{u}_{n+1}-3 \widehat{u}_{n}+3 \widehat{u}_{n-1}-\widehat{u}_{n-2} .
$$


It remains to bound the three terms on the right hand side of this inequality. The bound for the third term is given by Lemma 5.1:

$$
\left|\widehat{U}\left(t_{n}-t\right)-u\left(t_{n}-t\right)\right| \leq \frac{35 h^{4}}{1152}\left\|u^{(4)}\right\|_{*},
$$

and the term $\left|\varepsilon_{n-j}\right|$ can be bounded using Lemmas 5.2 and 5.3:

$$
\max _{0 \leq j \leq N_{T}}\left|\varepsilon_{j}\right| \leq C_{A}\left\{\frac{1}{12}+C_{B}\right\}\left\|u^{(4)}\right\|_{*} h^{4} .
$$

All of the basis functions $\phi_{j}$ are non-negative apart from $\phi_{1}(t)$, whose minimum value is $\phi_{1}(0)=-1 / 3$. Hence,

$$
\begin{aligned}
\sum_{j=0}^{n}\left|\varepsilon_{n-j}\right|\left|\phi_{j}(t / h)\right| & =\left(\left|\phi_{1}(t / h)\right|-\phi_{1}(t / h)\right)\left|\varepsilon_{n-1}\right|+\sum_{j=0}^{n}\left|\varepsilon_{n-j}\right| \phi_{j}(t / h) \\
& \leq\left(\frac{2}{3}+\sum_{j=0}^{n} \phi_{j}(t / h)\right) \max _{0 \leq j \leq N_{T}}\left|\varepsilon_{j}\right| \\
& \leq \frac{5 h^{4}}{3} C_{A}\left\{\frac{1}{12}+C_{B}\right\}\left\|u^{(4)}\right\|_{*} .
\end{aligned}
$$

The term $R_{n}$ can be bounded in a similar way to $R_{n}^{1}-R^{1}{ }_{n-1}$ in Lemma 5.3. The divided difference identity $f\left(t_{n+1}\right)-3 f\left(t_{n}\right)+3 f\left(t_{n-1}\right)-f\left(t_{n-2}\right)=h^{3} \int_{-3 / 2}^{3 / 2} B_{2}(s) f^{(3)}\left(t_{n-1 / 2}+s h\right) d s$, in terms of the quadratic B-spline $B_{2}(s)$ gives

$$
\widehat{u}_{n+1}-3 \widehat{u}_{n}+3 \widehat{u}_{n-1}-\widehat{u}_{n-2}=h^{3} \int_{-5 / 2}^{5 / 2} b_{2}(s) u^{(3)}\left(t_{n-1 / 2}+s h\right) d s,
$$

where $b_{2}(s)=\left(8 B_{2}(s)-B_{2}(s-1)-B_{2}(s+1)\right) / 6$, and so,

$$
\begin{gathered}
\left|\widehat{u}_{n+1}-3 \widehat{u}_{n}+3 \widehat{u}_{n-1}-\widehat{u}_{n-2}\right| \leq h^{3} \int_{-5 / 2}^{5 / 2}\left|b_{2}(s)\right|\left|u^{(3)}\left(t_{n-1 / 2}+s h\right)\right| d s \\
=h^{3}\left|u^{(3)}\left(\zeta_{n}\right)\right| \int_{-5 / 2}^{5 / 2}\left|b_{2}(s)\right| d s=h^{3}\left|u^{(3)}\left(\zeta_{n}\right)\right| \frac{516+11 \sqrt{11}}{450},
\end{gathered}
$$


for some $\zeta_{n} \in\left(t_{n-3}, t_{n+2}\right)$. Combining these three terms gives the bound

$$
\left|U_{n}\left(t_{n}-t\right)-u\left(t_{n}-t\right)\right| \leq C_{E}\left\|u^{(4)}\right\|_{*} h^{4}+C_{F}\left\|u^{(3)}\right\|_{*} B_{3}(t / h+1) h^{3}
$$

which yields the bound (5.11). The final bound follows from noting that $B_{3}(t / h-n-1)=0$ for $t \leq t_{n-1}$, and its maximum value for $t \in\left(t_{n-1}, t_{n}\right]$ is $1 / 6$.

Note that, in order to obtain an $\mathcal{O}\left(h^{4}\right)$ approximation over the whole range $t \in[0, T]$ where $T=N_{T} h$ involves running the scheme for one extra step to $n=N_{T}+1$.

6. Conclusions. The convolution spline scheme $(2.11)-(2.12)$ is a fourth order accurate approximation of the VIE (1.1) for general piecewise smooth (continuous or discontinuous) kernels which is efficient and straightforward to implement. The weights $q_{j}$ involve integrals of the kernel function multiplied by B-splines (or combinations of B-splines when near $t=0$ ); these can be evaluated to high accuracy by standard quadrature, and discontinuities in the kernel do not present any extra difficulties. This is not the case for methods such as convolution quadrature which rely on calculations in the Laplace domain.

Although much improved from [5], the regularity assumptions needed for the proof of Theorem 5.4 may not be optimal; the method appears stable and fourth order accurate for an even broader range of discontinuous kernels and forcing terms $a(t)$ than discussed here.

The numerical experiments in [5] indicate that the convolution spline method performs well for time domain boundary integral equations, and we are investigating whether the present analysis can be extended to these problems.

\section{REFERENCES}

1. L. Banjai and Ch. Lubich, An error analysis of Runge-Kutta convolution quadrature, BIT 51 (2011), 483-496.

2. L. Banjai and S. Sauter, Rapid solution of the wave equation in unbounded domains, SIAM J. Numer. Anal. 47 (2008), 227-249.

3. H. Brunner, Collocation methods for Volterra integral and related functional equations, Cambridge University Press, Cambridge, 2004.

4. G. Da Prat, Well test analysis for fractured reservoir evaluation, Elsevier, New York, 1990. 
5. P.J. Davies and D.B. Duncan, Convolution spline approximations of Volterra integral equations, J. Int. Equat. Appl. 26 (2014), 369-410.

6. C. De Boor, A practical guide to splines, Springer-Verlag, New York, 1978.

7. C.L. Epstein, L. Greengard and T. Hagstrom, On the stability of time-domain integral equations for acoustic wave propagation, Discr. Cont. Dynam. Syst. 36 (2016), 4367-4382.

8. G.C. Evans, Volterra's integral equation of the second kind, with discontinuous kernel, second paper, Trans. Amer. Math. Soc. 12 (1911), 429-472.

9. R.N. Horne, Modern Well test analysis: A computer-aided approach, Second edition, Petroway, Inc., Palo Alto, 1995.

10. F.J. Kuchuk, M. Onur and F. Hollaende, Pressure transient formation and well testing: Convolution, deconvolution and nonlinear estimation, Elsevier, New York, 2010.

11. Ch. Lubich, Convolution quadrature and discretized operational calculus, I, Numer. Math. 52 (1988), 129-145.

12. E. Messina, E. Russo and A. Vecchio, Convergence of solutions for two delays Volterra integral equations in the critical case, Appl. Math. Lett. 23 (2010), $1162-1165$.

13. Comparing analytical and numerical solution of a nonlinear twodelay integral equations, Math. Comp. Simu. 81 (2011), 1017-1026.

14. I. Muftahov, A. Tynda and D. Sidorov, Numerical solution of Volterra integral equations of the first kind with discontinuous kernels, arXiv:1507.06484v1, 2015.

15. M.J.D. Powell, Approximation theory and methods, Cambridge University Press, Cambridge, 1981.

16. A. Quarteroni and A. Valli, Numerical approximation of partial differential equations, Springer, New York, 1997.

17. S. Sauter and A. Veit, Retarded boundary integral equations on the sphere: exact and numerical solution, IMA J. Numer. Anal. 34 (2013), 675-699.

18. N.A. Sidorov and D.N. Sidorov, On the solvability of a class of Volterra operator equations of the first kind with piecewise continuous kernels, Math. Notes 96 (2014), 811-826.

19. E. Süli and D. Mayers, An introduction to numerical analysis, Cambridge University Press, Cambridge, 2003.

University of Strathclyde, Department of Mathematics and Statistics, 26 Richmond St., Glasgow, G1 1XH, UK

Email address: penny.davies@strath.ac.uk

Heriot-Watt University, Maxwell Institute for Mathematical Sciences, Department of Mathematics, Edinburgh, EH14 4AS, UK

Email address: D.B.Duncan@hw.ac.uk 